\title{
Using a Distributed Shapley-value Based Approach to Ensure Navigability in a Social Network of Smart Objects
}

\author{
Leonardo Militano*, Michele Nitti**, Luigi Atzori**, Antonio Iera* \\ *University of Reggio Calabria, Italy, leonardo.militano, antonio.iera@unirc.it \\ **University of Cagliari, Italy, michele.nitti, 1.atzori@diee.unica.it
}

\begin{abstract}
The huge number of nodes that is expected to join the Internet of Things in the short term will add major scalability issues to several procedures. A recent promising approach to these issues is based on social networking solutions to allow objects to autonomously establish social relationships. Every object in the resulting Social IoT (SIoT) exchanges data with its friend objects in a distributed manner to avoid the need for centralized solutions to implement major functionalities, such as: node discovery, information search and trustworthiness management. However, the number and types of established friendship affects network navigability. This paper addresses this issue proposing an efficient, distributed and dynamic strategy for the objects to select the right friends for the benefit of the overall network connectivity. The proposed friendship selection model relies on a Shapley-value based algorithm mapping the friendship selection process in the SIoT onto the coalition formation problem in a corresponding cooperative game. The obtained results show that the proposed solution is able to ensure global navigability, measured in terms of average path length among two nodes in the network, by means of a distributed and wise selection of the number of friend objects a node has to handle.
\end{abstract}

Index Terms-IoT, Shapley-value, Social Network, Network Navigability, Game Theory

\section{INTRODUCTION}

With the spreading of RFID-tagged objects and smart devices such as phones, TVs, and wireless sensor networks, the distance between physical and virtual worlds will be gradually shortened, leading to the so-called Internet of Things (IoT) paradigm. According to [1], by 2015 the RFID devices alone will reach the number of hundreds of billions; in line with this forecast, Cisco [2] foresees 6.58 connected smart devices per person by 2020 .

This large number of heterogeneous and pervasive objects continuously generate data about their sensed surroundings [3] and connect different realities, ranging from transport to education and from business to home management. Many applications can be built by exploiting information and services provided by groups of objects with different kinds of information about the physical world. However, this calls for effective methodologies for a fast and dynamic discovery of objects offering the desired service.

Indeed, as explained in [4], searching in the IoT represents a crucial challenge: in addition to the size of the searching space, sensors are often required to produce data in realtime, which then corresponds to highly dynamics readings, as it may happen when tracking the position of an object or sensing humidity/temperature/presence in the surrounding environment.

An approach that has the potentials to properly address the mentioned scalability issues and that has been gaining popularity in the last years is based on the use of social networking technologies to allow objects to autonomously establish social relationships. The driving motivation is that a social-oriented approach is expected to put forward the discovery, selection and composition of services and information provided by distributed objects and networks that have access to the physical world [5], [6] and [7]. In [8] the Social IoT (SIoT) concept is formalized, which is intended as a social network where every node is an object capable of establishing social relationships with other things in an autonomous way according to rules set by the owner. Then, every object can look for the desired service by exploiting the knowledge about its friendships to guarantee an efficient and scalable search for services and objects in the same way humans seek for information in social networks. Accordingly, the resulting searching process is distributed, as each object that needs to find the target peer looks among its friends, which in turn inquire their friends if they cannot provide the requested service.

The belief that objects would be able to navigate the SIoT network with only local information is founded on the works of the sociologist Stanley Milgram [9] and the computer scientist Jon Kleinberg [10]. Milgram studied the small-world phenomenon and demonstrated that people are tied by short chains of acquaintances, while Kleinberg concluded that there are structural clues in a social network that help people to efficiently find a short path even without a global knowledge of the network. Clearly, the resulting SIoT performance is strictly subject to the capability of the objects to replicate the human innate behavior when handling social relationship, e.g., select the right friends, consult the appropriate service provider, evaluate the trustworthiness of the peers and community. To this aim, each object has to store and manage the information related to its friends, data about past transactions, quality of services for past interactions, services that can be provided by friends, and so on. An important parameter that needs to be considered is the number of relationships that an object establishes, which affects its memory consumption, the waste 
of computational power and battery, and the effectiveness of service discovery operations. Which object to promote as a friend among the potential candidates is also a key aspect for the overall system performance.

These issues have been recently addressed; however the proposed strategies are quite simple and the performance has only been analyzed in terms of global [11] and local [12] network navigability. These considerations motivate the present paper, which aims at defining an efficient strategy for the objects to select the right friends for the benefit of the overall network navigability. An important feature that has to be guaranteed is that the resulting friend selection policy has to be distributed and dynamic so that no central controllers have to set a-priori static rules to establish social ties. Additionally, the navigability of the resulting network structure should be independent on the implemented routing algorithm and the application triggering the search operations. In this view, the major contributions of the paper are the following:

- We define a model for friendship selection that rely on a Shapley-value based algorithm. The utility function carefully models the corresponding game to meet the posed constraints.

- We evaluate the performance of network navigability in terms of average number of hops by using local peer search operations.

The remainder of the paper is organized as follows. In Section II the proposed game theoretic solution for the problem is described, whereas Section III reports on the details of the proposed friendship selection algorithm. The performance evaluation results are summarized in section IV, and conclusive remarks are given in the last section.

\section{Proposed Approach for Friendship Selection in the SIoT}

How to select an effective set of friendship relationships among the possible candidates has been addressed in the past in the context of information diffusion in social networking problems. Usually, a score is assigned to each member of the set of potential devices [13], where the selected score somehow corresponds to the importance of that device for the application at hand. However, such a conventional approach suffers from the main intrinsic limitation that it only considers the relative importance of objects as stand-alone entities. Contrarily, the key requirement is to understand the importance of each object in terms of its contribution to a certain utility when combined with other nodes [14]. The flexibility, which comes from the ability to take into account the contributions of all possible combinations of objects (rather than considering just one node at a time), is absent in conventional centrality measures. This is a crucial limitation in many applications and represents the reason why game theoretic network centrality measures have been proposed in research activities relevant to social networks of humans.

Solutions to the raised problem can be effectively found by using one of the most used concepts in value or cost-sharing game theoretic applications, namely the Shapley-value [15]. Thanks to its intrinsic capability to capture the contribution of the single players to different coalitions of players, the Shapley-value has found several applications both in networking and social analysis. For instance in [16] it is adopted for a monetary cost analysis for a fair content sharing for both network/service providers and the end-users. In the domain of social networks and networks in general, the Shapley-value is adopted as a measure of the relative importance of single nodes [17]. Closer to the contribution of this paper, the Shapley-value has been applied to social networking problems in [18], [13] and [19]. In particular, in [18] a set of new centrality measures are proposed based on cooperative game theoretic notions. An analysis of the Shapley-value for network centrality has been presented also in [13], with results demonstrating the opportunities for efficiency gains. In [19] the focus is on the information diffusion problem in social networks with particular focus on the target set selection issue.

These promising results obtained for the information diffusion problem in social networking of humans suggest that the Shapley-value has interesting features that can also be exploited for the friendship selection problem in the Social Internet of Things addressed by our paper. We then propose a Shapley-value based game theoretic modeling for friendship selection in the SIoT. In particular, we map the friendship selection process in the SIoT onto the coalition formation problem in a corresponding cooperative game. The Shapleyvalues of the nodes in the game represents the importance of an object in the social IoT network and will be used to set the friendship preferences. The adopted utility function plays a key role for the problem and will be carefully modeled for the corresponding game to meet the posed constraints. In the following section we will provide an overview of Social Internet of Things concepts.

\section{A. Social Internet of Things}

In this paper, without losing of generality, we refer to the social IoT model proposed in [8] (we use the acronym SIoT to refer to it). According to this model, a set of forms of socialization among objects are foreseen. The parental object relationship is defined among similar objects, built in the same period by the same manufacturer (the role of family is played by the production batch). Moreover, objects can establish co-location object relationship and cowork object relationship, like humans do when they share personal (e.g., cohabitation) or public (e.g., work) experiences. A further type of relationship is defined for objects owned by the same user (mobile phones, game consoles, etc.) that is named ownership object relationship. The last relationship is established when objects come into contact, sporadically or continuously, for reasons purely related to relations among their owners (e.g., devices/sensors belonging to friends); it is named social object relationship. These relationships are created and updated on the basis of the objects features (such as: object type, computational power, mobility capabilities, brand) and activity (frequency in meeting the other objects, mainly). The management of the relationships is implemented in the cloud, in the object gateways, and in objects themselves 
if capable of implementing the relevant logic. Clearly, the configuration of these functionalities is controlled by the object owner; accordingly, the resulting links are asymmetrical.

Before going into the details of the proposed approach, in the next section we will first recall some basic notions of game theory used in this paper. For more information on the subject, the reader is referred to the vast literature available, such as for example [20].

\section{B. Game-Theoretic Notions for Network Navigability}

The process of assigning a value to each node/object in a network can be naturally modeled as a coalitional game $G=\langle\mathcal{N}, v(\cdot)\rangle$ with transferable utilities (TU), where $\mathcal{N}$ is the set of $\mathrm{N}$ players and $v(\cdot)$ is a value function [21]. A value function $v(\mathcal{S})$ is a mapping from nonempty coalition $\mathcal{S}$ to a real number. Thus, given a subset $\mathcal{S}$ of $\mathcal{N}$, we call $v(\mathcal{S})$ the value of the coalition $\mathcal{S}$, which is the maximum aggregated payoff available for division among players who are members of $\mathcal{S}$ when working together without the help of players in $\mathcal{N} \backslash \mathcal{S}$. The set of players $\mathcal{N}$ is called the grand coalition and $v(\mathcal{N})$ is called the value of the grand coalition.

We can define $x \in \mathfrak{R}^{|\mathcal{N}|}(|\cdot|$ represents the cardinality of a set) as a vector one-to-one associated with players in $\mathcal{N}$, so that $x_{i}$ denotes the component associated with player $i \in \mathcal{N}$. For any vector $x \in \mathfrak{R}^{|\mathcal{N}|}$, we denote by $x(\mathcal{S})$ the value $\sum_{i \in \mathcal{S}} x_{i}$, where $\mathcal{S} \subseteq \mathcal{N}$ is a coalition. A feasible payoff profile (or preimputation) of $G$ is a vector $x \in \mathfrak{R}^{|\mathcal{N}|}$ such that $x(\mathcal{N})=v(\mathcal{N})$. An imputation of $G$ is a feasible value profile $x \in \mathfrak{R}^{|N|}$ such that $x_{i} \geq v(\{i\})$, for each $i \in \mathcal{N}$. This condition is usually called individual rationality. The set of all imputations of the game $G$ is denoted by $X(G)$. An outcome for $G$ is an imputation from $X(G)$ that specifies the distribution of the value to any player of the game. A typical requirement of a good outcome is to be "stable" with respect to the possibility that subsets of players find convenient to deviate from it and form alternative coalitions to attain higher values. The set of such stable outcomes is known as the core of the game.

There are many solution concepts proposed in Game Theory literature, but only those associated with unique profiles are usually desirable in practical applications. Among these, the Shapley-value [15] is one of the most used. In particular, it describes an effective approach to the fair allocation of gains obtained from the cooperation among players of a cooperative game. Since some players may contribute more to the total value than others, an important requirement is to fairly distribute gains among the players. To this purpose, in deciding the payoff to be allocated to the players, the Shapleyvalue accounts for the relative importance of each player to the game.

Definition 1 (Shapley-value [15]). The Shapley-value of a TU game $G=\langle\mathcal{N}, v(\cdot)\rangle$ is the pre-imputation of $G$ assigning to every player $i \in \mathcal{N}$ the following value

$$
\phi_{i}(G)=\frac{1}{|\mathcal{N}| !} \sum_{\mathcal{S} \subseteq \mathcal{N} \backslash\{i\}}|\mathcal{S}| !(|\mathcal{N}|-|\mathcal{S}|-1) ![v(\mathcal{S} \cup\{i\})-v(\mathcal{S})] .
$$

In words, the Shapley-value assigns a value to each player $i$ by taking into account his average marginal contribution, where the average is computed over all different sequences according to which the grand coalition could be built up from the empty coalition. In general, the Shapley-value may fall outside the core, even if the core is not empty. Thus, in particular, the Shapley-value is not necessarily an imputation, and it may violate the individual rationality condition.

This solution concept has also a nice axiomatic characterization supporting its notion of fairness (it is the unique preimputation that satisfies the Symmetry, Dummy Player, and Additivity axioms). A natural way to interpret the Shapleyvalue $\phi_{i}(G)$ of player $i$ is in terms of the average marginal contribution that player $i$ makes to any sub-coalition of $\mathcal{N}$ assuming all orderings are equally likely. The Shapley-value takes into account all possible coalitional dynamics and negotiation scenarios among the players and comes up with a single unique way of distributing the value $v(\mathcal{N})$ of the grand coalition among all the players. Thus, the Shapley-value of a player accurately reflects the bargaining power of the player and the marginal value the player brings to the game.

An additional simplifying assumption, usually made when modeling a TU coalitional game, is that the game is superadditive. A game is superadditive if the characteristic function of the game is such that for any $\mathcal{S}$ and $\mathcal{T}$ disjoint coalitions $(\mathcal{S} \cap \mathcal{T}=\emptyset)$ subsets of $\mathcal{N}$, we have that $v(\mathcal{S} \cup \mathcal{T}) \geq v(\mathcal{S})+v(\mathcal{T})$. In words, a coalition formed by the merger of two or more coalitions will realize a value at least as great as the sum of the values of the coalitions merged. This seems a reasonable assumption for coalitional games and the proposed Shapleyvalue is usually applied in superadditive games. However, the Shapley-value can also be computed for games with a non superadditivite characteristic function [22]. In these cases the Shapley-value will lack the property of individual rationality, thus, some players may have values less than the value they can generate as a singleton coalition. Noteworthy, in this paper the game model does not foresee a value to be shared among players, but it is used to find the most influential objects among a set of candidate friend objects. Therefore, also non superadditive characteristic functions are acceptable for our scope.

\section{FRIENDSHIP SELECTION ALGORITHM}

For simplicity in the analysis, we assume that the objects in the SIoT have a commonly shared criterion to decide whether to consider another object as a candidate object for a friendship relation; this criterion is symmetric. For instance, if an object $i$ meets the given criterion with another object $j$, then also node $j$ meets the criterion w.r.t. node $i$. Moreover, we assume that each object can establish up to a maximum number of friendships $N_{\max }$, as each object has limited resources. When the criterion is met between a couple of objects not friends yet, then a new friendship is directly established if the list of friends for the two nodes has less than $N_{\max }$ objects. If this is not the case, then a friendship selection algorithm is 
triggered to choose the most influential $N_{\max }$ friends among the candidate objects.

The proposed friendship selection algorithm aims at selecting the objects that offer the maximum marginal contribution to the global services in the SIoT. To compute the marginal contributions of the candidate objects, a cooperative coalitional game, as described in the previous section, is modeled. The set of candidate objects are the players in the cooperative game and the Shapley-value is used to compute the marginal contributions of the individual players to the overall value achieved by the grand coalition in the game. First, based on the Shapley-value, a ranking list of the candidate objects is computed, and then the top $N_{\max }$ objects are selected, with the only constraint that a node cannot refuse or discard relationships if this action is going to isolate a node. If nodes $i$ and $j$ are mutually in the top $N_{\max }$ objects of the respectively computed ranking lists, the friendship is established. In some cases the establishment of a new friendship may require an old one to be removed. When this happens, the choice of the friendship to be removed is again driven by the Shapley-value based ranking list.

A key aspect for the success of the proposed algorithm is the wise definition of the utility function, which measures the contribution of the candidate objects. By focusing on an object $i$, which has to determine its $N_{\max }$ friendships, we will next define an utility function that best meets the system requirements.

\section{A. Average local clustering based model}

The utility function we consider for the model is based on the average local clustering coefficient [23]. This is defined as: $\bar{C}=\frac{1}{n} \sum_{j=1}^{n}$ Clocal $_{j}$ where $n$ is the number of players in a coalition and Clocal $_{j}$ is the local clustering coefficient defined as: Clocal $_{j}=\frac{2 \cdot e_{j}}{k_{j} \cdot\left(k_{j}-1\right)}$, where $k_{j}$ is the number of neighbors for node $j$ and $e_{j}$ is the number of edges among them. The driving motivation comes from Kleinberg's findings [10], namely that network navigability is assured by the ability to reach nodes with low values of local clustering since they are hardly reachable through other paths. This gives us the possibility of performing local choices that will guarantee that the global navigability of the network is kept at acceptable levels.

The Transferable utility (TU)-game $G=\langle\mathcal{N}, v(\cdot)\rangle$ is modeled on the set of $N_{\text {max }}+1$ candidate friends for node $i$ plus the node $i$ itself, thus we have that $|\mathcal{N}|=N_{\text {max }}+2$. This number derives from the consideration that the selection algorithm is triggered only when a new object meets a given criterion to become a friend of object $i$ and this additional object would make the number of friends go beyond the threshold $N_{\text {max }}$. Moreover, also node $i$ must be considered, as the utility function is directly influenced by the presence of node $i$ in any of the coalitions of objects. In details, we define the value function as:

$$
v(\mathcal{S})=\left(1-\frac{1}{|\mathcal{S}|} \sum_{i \in \mathcal{S}} \text { Clocal }_{i}\right) \forall \mathcal{S} \subseteq \mathcal{N}, \text { and } v(\emptyset)=0
$$

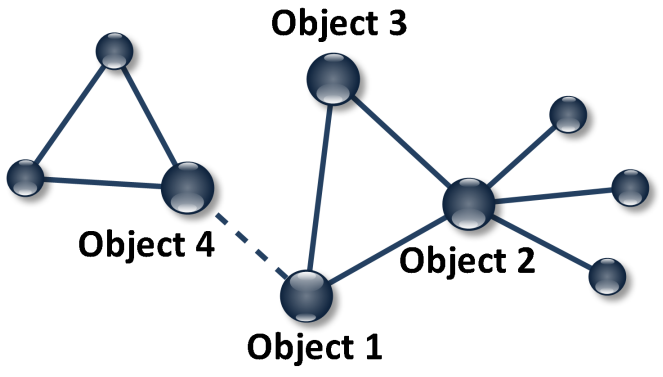

Fig. 1. Friendship selection based on local clustering sample scenario.

Noteworthy, this utility function is non superadditive as in some topology configurations some nodes, which join a preexisting set of friends, may actually adversely affect the local clustering coefficient value.

We then sort the players in decreasing order of their Shapley-value. From the so-constructed rank list, the candidate objects are selected one at the time by scrolling the list in a top-down order (clearly, node $i$ itself will not be considered as a candidate friend). If the friendship with an object is already active, then nothing happens and the subsequent node in the list is selected until $N_{\max }$ nodes are selected from the list. If the selected object has not been considered as a friend yet, then the node tries to form a new friendship relationship. If the new friendship request is accepted, then an old friendship must be closed to meet the constraint on the $N_{\max }$ number of friendships of node $i$. Also for this choice the Shapley-based rank list is used, and the less influential node is selected for removal.

To better understand the behavior of the proposed solution, let us consider the sample study case shown in Fig. 1. In particular, objects 1 and 4 meet the criterion for a new potential friendship (see dashed line in the Figure) and the proposed algorithm is triggered to possibly update the friendships for the involved objects. As an example, let us focus the attention on object 1 and the computation of its list of preferences for the friendships (a similar analysis can be repeated for object 4) in the case where the maximum number of friends per object 1 is set to $N_{\max }=2$. This means that the two objects with the highest Shapley-value shall be selected as its friends. In the scenario of the figure we can model the game as follows: $\mathcal{N}=$ $\{1,2,3,4\} ; v(1)=v(4)=v(14)=0.667 ; v(2)=0.9 ; v(3)=$ $0 ; v(12)=v(24)=1-1 / 2 \cdot(0.333+0.1)=0.783 ; v(13)=$ $v(34)=1-1 / 2 \cdot(0.333+1)=0.333 ; v(23)=1-1 / 2 \cdot(0.1+$ 1) $=0.45 ; v(123)=v(234)=1-1 / 3 \cdot(0.333+0.1+1)=$ $0.522 ; v(124)=1-1 / 3 \cdot(0.333+0.1+0.333)=0.744 ; v(134)=$ $1-1 / 3 \cdot(0.333+1+0.333)=0.444 ; v(1234)=1-1 / 4 \cdot$ $(0.333+0.1+1+0.333)=0.558$. By computing the marginal contributions of the players based on the Shapley-value we obtain the results in Table I. We observe that the order of preferred friendships for object 1 are respectively, object 2, 
object 4 and object 3 . Since $N_{\max }=2$, object 1 will try to form a friendship with objects 2 and 4 first, and consider object 3 only if any of the preferred friendships is not accepted by the inquired objected.

TABLE I

ShaPLEY-VALUE FOR SAMPLE SCENARIO IN Fig. 1.

\begin{tabular}{|c|c|c|c|c|}
\hline ase & Object 1 & Object 2 & Object 3 & Object 4 \\
\hline$\overline{1234}$ & $\bar{v}(1)-v(\emptyset)=0.667$ & $\bar{~} \bar{v}(12)-v(1)=0.116$ & 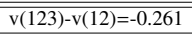 & $\bar{c}(\mathcal{N})-\mathrm{v}(123)=0.036$ \\
\hline 1243 & $v(1)-v(\emptyset)=0.667$ & $v(12)-v(1)=0.116$ & $\mathrm{v}(\mathcal{N})-\mathrm{v}(124)=-0.186$ & $v(124)-v(12)=-0.039$ \\
\hline 1324 & $v(1)-v(\emptyset)=0.667$ & $\mathrm{v}(123)-\mathrm{v}(13)=0.189$ & $v(13)-v(1)=-0.333$ & $\mathrm{v}(\mathcal{N})-\mathrm{v}(123)=0.036$ \\
\hline 1342 & $v(1)-v(\emptyset)=0.667$ & $v(\mathcal{N})-v(1)$ & $=-0.333$ & $\mathrm{v}(134)-\mathrm{v}(13)=0.111$ \\
\hline 1423 & $\mathrm{v}(1)-\mathrm{v}(\emptyset)=0.667$ & $\mathrm{v}(124)-\mathrm{v}(14)=0.077$ & $v(\mathcal{N})-v(124)=-0.186$ & $\mathrm{v}(14)-\mathrm{v}(1)=0$ \\
\hline 1432 & $v(1)-v(\emptyset)=0.667$ & $\mathrm{v}(\mathcal{N})-\mathrm{v}(134)=0.114$ & $\mathrm{v}(134)-\mathrm{v}(14)=-0.222$ & $\mathrm{v}(14)-\mathrm{v}(1)=0$ \\
\hline 2134 & $v(12)-v(2)=$ & $v(2)-v$ & $\mathrm{v}(123)-\mathrm{v}(1$ & $\mathrm{v}(\mathcal{N})-\mathrm{v}$ \\
\hline 2143 & $v(12)-v(2)=-0.117$ & $\mathrm{v}(2)-\mathrm{v}(\emptyset)=0.9$ & $\mathrm{v}(\mathcal{N})-\mathrm{v}(124)=-0.186$ & $\mathrm{v}(124)-\mathrm{v}(12)=-0.039$ \\
\hline 2314 & $v(123)-v(23)=0.072$ & $\mathrm{v}(2)-\mathrm{v}(\emptyset)=0.9$ & $v(23)-v(2)=-0.45$ & $\mathrm{v}(\mathcal{N})-\mathrm{v}(123)=0.036$ \\
\hline 2341 & $\mathrm{v}(\mathcal{N})-\mathrm{v}(234)=0.036$ & $\mathrm{v}(2)-\mathrm{v}(\emptyset)$ & $v(23)-v(2)=-0.45$ & $\mathrm{v}(234)-\mathrm{v}(23)=0.072$ \\
\hline 2413 & $\mathrm{v}(124)-\mathrm{v}(24)=-0.039$ & $\mathrm{v}(2)-\mathrm{v}(\emptyset)=0.9$ & $\mathrm{v}(\mathcal{N})-\mathrm{v}(124)=-0.186$ & $\mathrm{v}(24)-\mathrm{v}(2)=-0.117$ \\
\hline 2431 & $\mathrm{v}(\mathcal{N})-\mathrm{v}(23$ & $v(2)-v$ & $\mathrm{v}(234)-\mathrm{v}(24)=-0.261$ & $=-0.117$ \\
\hline 3124 & $\mathrm{v}(13)-\mathrm{v}(3)=0.333$ & $\mathrm{v}(123)-\mathrm{v}($ & $\mathrm{v}(3)-\mathrm{v}(\emptyset)=0$ & $\mathrm{v}(\mathcal{N})-\mathrm{v}(123)=0.036$ \\
\hline 3142 & $v(13)-v(3)=0.333$ & $\mathrm{v}(\mathcal{N})-\mathrm{v}(134)=0.114$ & $v(3)-v(\emptyset)=0$ & $\mathrm{v}(134)-\mathrm{v}(13)=0.111$ \\
\hline 3214 & $\mathrm{v}(123)-\mathrm{v}(23$ & $\mathrm{v}(23)-\mathrm{v}$ & $\mathrm{v}(3)-$ & $\mathrm{v}(\mathcal{N})-\mathrm{v}(123)=0.036$ \\
\hline 3241 & $\mathrm{v}(\mathcal{N})-\mathrm{v}(2$ & $v(23)-v$ & $\mathrm{v}(3)$ & $\mathrm{v}(234)-\mathrm{v}(23)=0.072$ \\
\hline 3412 & $\mathrm{v}(134)-\mathrm{v}($ & $\mathrm{v}(\mathcal{N})-\mathrm{v}(1$ & $v(3)-$ & $v(34)-v(3)=0.333$ \\
\hline 3421 & $\mathrm{v}(\mathcal{N})-\mathrm{v}(2$ & $v(234)-v(34)=0$ & $v(3)-$ & $v(34)-v(3)=0.333$ \\
\hline 4123 & $\mathrm{v}(14)-$ & $v(124)-\mathrm{v}$ & $\mathcal{N})-\mathrm{v}(1$ & $\mathrm{v}(4)-$ \\
\hline 4132 & $v(14)-v$ & $\mathrm{v}(\mathcal{N})-\mathrm{v}(134)=0.114$ & $\mathrm{v}(134)-\mathrm{v}($ & $v(4)-v(\emptyset)=0.667$ \\
\hline 4213 & $v(124)-v(24)=-0.039$ & $\mathrm{v}(24)-\mathrm{v}(4)=0.117$ & $\mathrm{v}(\mathcal{N})-\mathrm{v}(124)=-0.186$ & $v(4)-v(\emptyset)=0.667$ \\
\hline 4231 & $\mathrm{v}(\mathcal{N})-\mathrm{v}(234)=0.036$ & $\mathrm{v}(24)-\mathrm{v}(4)=0.117$ & $\mathrm{v}(234)-\mathrm{v}(24)=-0.261$ & $v(4)-v$ \\
\hline 4312 & $\mathrm{v}(134)-\mathrm{v}(34)$ & $\mathrm{v}(\mathcal{N})-\mathrm{v}(134)=0.114$ & $v(34)-v(4$ & $\mathrm{v}(4)-\mathrm{v}(\emptyset)=0.6$ \\
\hline 4321 & $\mathrm{v}(\mathcal{N})-\mathrm{v}(234)=0.036$ & $v(234)-v(34)=0.189$ & $\mathrm{v}(34)-\mathrm{v}(4)=-0.333$ & $v(4)-v(\emptyset)=0.667$ \\
\hline$\overline{\phi_{i}}$ & 0.2058 & 0.348 & -0.202 & 0.2058 \\
\hline
\end{tabular}

\section{Performance Evaluation}

A numerical evaluation has been conducted by using the MATLAB $^{\circledR}$ tool for a wide set of scenarios, to observe the achievable performance in terms of navigability of the Social IoT. In doing so, information about new relationship requests received by the objects (based on their profile, settings, and movements) is needed for huge numbers of real objects. Unfortunately, this data is not available to date, as real applications involving a high number of Social Things have not been deployed yet. We thus rely on the Barabási-Albert model [24], which is able to generate scale-free networks based on preferential attachment with the same characteristics of real social networks.

The maximum number of friendship relationships $N_{\max }$ can (i) either be fixed a-priori, (ii) or vary during the network lifetime. The second option helps to keep under control the number of hubs in the network, which influences the network navigability. In particular, for this case the value for $N_{\max }$ is increased when

$$
\begin{aligned}
& \text { there are } x \% \text { of the } N \text { nodes in the network with at } \\
& \text { least } y \% \text { of } N_{\max } \text { friends, }
\end{aligned}
$$

where $x$ represents the maximum percentage of hubs in the network, whereas $y$ represents the threshold for a node to become a hub. We study the behavior of the networks obtained with the Barabási-Albert model, with or without applying the proposed Shapley-based strategies. The attention will be on understanding when a node can be considered as a hub and how many hubs are necessary in a network to improve network navigability in terms of the average path length. To this aim, we consider a simple local routing approach. In particular, we refer to a scenario where a given object $\mathrm{A}$ wishes to

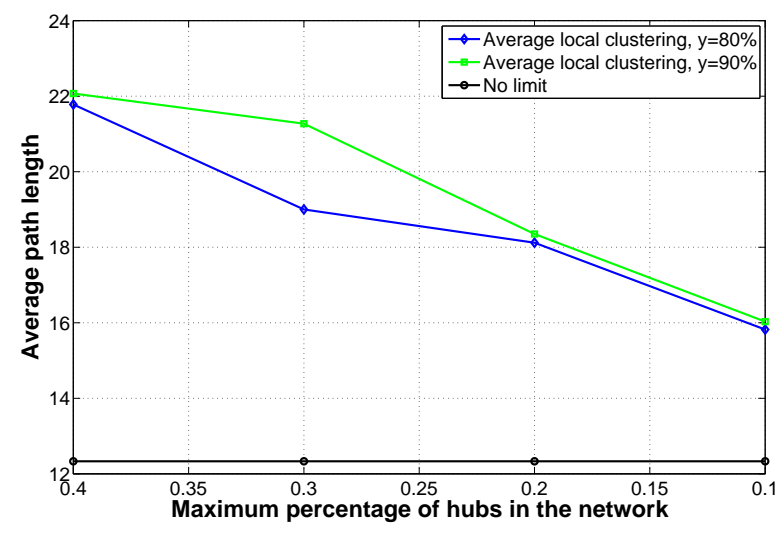

Fig. 2. Average path length for different values of the maximum percentage of hubs in the network.

communicate with node B. The first task to perform is to check whether it has a direct connection with object B, that is, B is among its friends. If not, $\mathrm{A}$ asks to the friend object with the highest connectivity degree, let say $\mathrm{X}$, to find a route to reach B. Then, object X repeats the same procedure until object B is finally reached.

In Fig. 2 the average path length is reported, for different values in the range $[0.1-0.4] \%$ of the maximum percentage of hubs $x$ in the network and considering either $y=80 \%$ or $y=90 \%$. The novel proposed solutions are also compared with the case where no strategy is applied (labeled as "no limit" in the plots). As we can observe, a reduction in the number of hubs in the network allows for an increase in the performance of the network, as suggested by Kleinberg. This effect can also be obtained when decreasing the threshold for a node to become a hub, i.e., the $y$-value. However, if we relax too much the control parameters, we fall again in the scenario "no limit". In fact, if $x$ is set to $\frac{1}{N} \%$ then for every node reaching a number of friends equal to $y \%$ of $N_{\max }$, the value of $N_{\max }$ increases. On the other hand, if we set $y=0 \%$, every node is considered as an hub and $N_{\max }$ increases no matter what the value for $x$ is.

The other important result investigated is the maximum number of friends reached by a node. This has been tested for several combination of $x$ and $y$, as plotted in Fig. 3. We can observe how the proposed algorithm is able to drastically decrease the number of friends that a node has to manage. This is a interesting results, as this has a positive effect on the computational burden for the objects. By comparing Fig. 2 and Fig. 3, an important observation is that if the number of hubs in the network is low then each hub has to manage more friends. This last feature means that by using more stringent values of the parameters $x$ and $y$, it is possible to achieve better performance in terms of local navigability, but this goes at the cost of an increase in the memory consumption, computational power and battery life. To limit this problem, an enhancement for the solution could be to adjust the maximum number of friendships $N_{\max }$ based on the node features and 


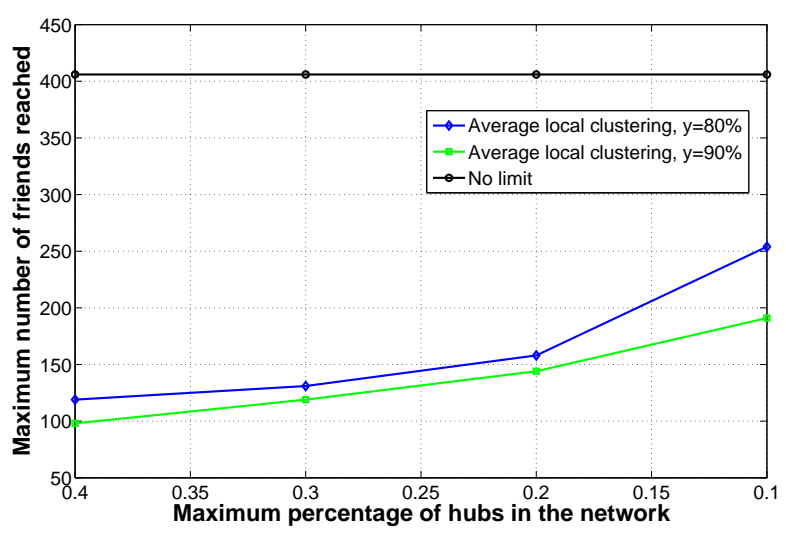

Fig. 3. Maximum number of friends for different values of the maximum percentage of hubs in the network.

then allow only nodes with high computation capabilities, such as vehicles or smart devices, to become hubs.

A further extension for the implementation of the proposed solution that will be considered in our future research, is to consider enhancing aspects referring to the average path length:

- in our simulations we have considered all the possible pairs of nodes to be uniformly distributed over the network; however, it has been proved that friends share similar interests (bringing to the homophily phenomenon [25]), so that it is highly probable to find another node in the friends list or in the friend of a friend (FOAF) list, thus reducing the average path length among all the pairs of nodes;

- node similarity for the routing operations has not been considered so far; indeed, in our simulations nodes try to reach their destination by using only information about the degree of their neighbors. However, external properties could be used to select the right nodes (among available friends) to which ask for the desired service.

\section{Conclusion}

In this paper we defined the problem of friendship selection in the SIoT, highlighting that if a Social Network of Things is properly created it can show the characteristics of a small world network and comply with the condition for network navigability. Based on this knowledge, we proposed a model for a distributed friendship selection that relies on the Shapleyvalue. To this aim, the friendship selection process in the SIoT is mapped onto the coalition formation problem for a corresponding cooperative game. Based on a proposed utility function, that meets the constraints for the system, the Shapley-value nicely models the importance of an object in the social IoT network and is used to set the friendship preferences. The resulting network navigability in terms of average number of hops by using local peer search operations is evaluated and compared with a standard solution where no limit on the friendships is set for the objects.

\section{REFERENCES}

[1] IDTechEx, Raghu Das, and Peter Harrop, RFID forecasts, players and opportunities 2011-2021, IDTechEx, 2011.

[2] Dave Evans, "The internet of things: how the next evolution of the internet is changing everything," CISCO white paper, vol. 1, 2011.

[3] L. Atzori, A. Iera, and G. Morabito, "The internet of things: A survey," Computer networks, vol. 54, no. 15, pp. 2787-2805, 2010.

[4] D. Zhang, L.T. Yang, and H. Huang, "Searching in internet of things: Vision and challenges," in Parallel and Distributed Processing with Applications (ISPA), 2011 IEEE 9th International Symposium on, 2011, pp. 201-206.

[5] A.J. Jara, P. Lopez, D. Fernandez, J.F. Castillo, M.A. Zamora, and A.F. Skarmeta, "Mobile digcovery: discovering and interacting with the world through the internet of things," Personal and Ubiquitous Computing, vol. 18 , no. 2, pp. 323-338, 2014.

[6] P. Mendes, "Social-driven internet of connected objects," in Proc. of the Interconn. Smart Objects with the Internet Workshop, 2011.

[7] M. Nitti, R. Girau, and L. Atzori, "Trustworthiness management in the social internet of things," Knowledge and Data Engineering, IEEE Transactions on, vol. 26, no. 5, pp. 1253-1266, May 2014.

[8] L. Atzori, A. Iera, G. Morabito, and M. Nitti, "The social internet of things (siot)-when social networks meet the internet of things: Concept, architecture and network characterization," Computer Networks, vol. 56, no. 16 , pp. 3594-3608, 2012

[9] J. Travers and S. Milgram, "An experimental study of the small world problem," Sociometry, pp. 425-443, 1969.

[10] J. Kleinberg, "The small-world phenomenon: An algorithmic perspective," in Proceedings of the thirty-second annual ACM symposium on Theory of computing. ACM, 2000, pp. 163-170.

[11] M. Nitti, L. Atzori, and IP. Cvijikj, "Network navigability in the social internet of things," in Internet of Things (WF-IoT), 2014 IEEE World Forum on, March 2014, pp. 405-410.

[12] M. Nitti, L. Atzori, and I.P. Cvijikj, "Friendship selection in the social internet of things: challenges and possible strategies," Internet of Things Journal, IEEE, vol. PP, no. 99, pp. 1-1, 2014.

[13] K.V. Aadithya, B. Ravindran, T.P. Michalak, and N.R. Jennings, "Efficient computation of the shapley value for centrality in networks," in Internet and Network Economics, pp. 1-13. Springer, 2010.

[14] D. Kempe, J. Kleinberg, and É. Tardos, "Influential nodes in a diffusion model for social networks," in Automata, languages and programming, pp. 1127-1138. Springer, 2005.

[15] L.S. Shapley, "A value for n-person games," Tech. Rep., DTIC Document, 1952.

[16] L. Militano, A. Iera, and F. Scarcello, "A fair cooperative content-sharing service," Computer Networks, vol. 57, no. 9, pp. 1955-1973, 2013.

[17] P. Papapetrou, A. Gionis, and H. Mannila, "A shapley value approach for influence attribution," in Machine Learning and Knowledge Discovery in Databases, pp. 549-564. Springer, 2011.

[18] D. Gómez, E. González-Arangüena, C. Manuel, G. Owen, M. del Pozo, and J. Tejada, "Centrality and power in social networks: a game theoretic approach," Mathematical Social Sciences, vol. 46, no. 1, pp. 27-54, 2003.

[19] R. Narayanam and Y. Narahari, "A shapley value-based approach to discover influential nodes in social networks," IEEE Transactions on Automation Science and Engineering, , no. 99, pp. 1-18, 2010.

[20] M.J. Osborne, An introduction to game theory, vol. 3, Oxford University Press New York, 2004.

[21] L. Militano, A. Iera, A. Molinaro, and F. Scarcello, "Energy-saving analysis in cellular wlan cooperative scenarios," Vehicular Technology, IEEE Transactions on, vol. 63, no. 1, pp. 478-484, Jan 2014.

[22] R.J. Aumann, "Some non-superadditive games, and their shapley values, in the talmud," International Journal of Game Theory, vol. 39, no. 1, pp. $3-10,2010$.

[23] D.J. Watts and S.H. Strogatz, "Collective dynamics of smallworldnetworks," nature, vol. 393, no. 6684, pp. 440-442, 1998.

[24] A.-L. Barabási and R. Albert, "Emergence of scaling in random networks," science, vol. 286, no. 5439, pp. 509-512, 1999.

[25] H. Bisgin, N. Agarwal, and Xiaowei Xu, "Investigating homophily in online social networks," in Web Intelligence and Intelligent Agent Technology (WI-IAT), 2010 IEEE/WIC/ACM International Conference on, 2010, vol. 1, pp. $533-536$. 